\title{
OPTIMIZATION WITH DIFFERENT REDUNDANT HYDRAULIC PIPELINES
}

\author{
Sihem BOURI*, Abdelhalim BENMANSOUR ${ }^{* *}$ \\ Materials and Renewable Energy Research Unit (URMER), \\ University Aboubekr Belkaid Tlemcen BP 119 - 13000, Algeria, \\ E-mail: *bouri_sihem@yahoo.fr, ${ }^{* *}$ halim.benmansour13@gmail.com
}

\begin{abstract}
This paper uses an ant system (AS) meta-heuristic optimization method to solve the problem of hydraulic structure optimization systems. We consider the case where redundant hydraulic pipelines are chosen to achieve a desirable level of reliability. The hydraulic pipelines of the system are characterized by their cost, capacity and reliability. The reliability is defined as the ability to satisfy the water consumer demand which is represented as a piecewise cumulative load curve. The proposed meta-heuristic seeks for the optimal configuration of series-parallel systems in which a multiple choice of hydraulic pipelines are allowed from a list of product available in the hydraulic market. Our approach has the advantage to allow hydraulic pipelines elements with different parameters to be allocated in hydraulic systems. To allow fast reliability estimation, a universal generating function method is applied.
\end{abstract}

Keywords: Ant colony, Optimization, Hydraulic, reliability, Universal moment generating Function (UMGF)

\section{INTRODUCTION}

Cost configuration of electrical power system structure is series-parallel. Elements of the system with different capacities and costs where considered, and demand was estimated using a load curve. The drawback of the approach adopted in [3] and [4] is that costs of elements are defined as explicit analytical function of their capacities and the same reliability index values are assigned to all the elements of given type, regardless of their capacity. In [2], a genetic algorithm approach is used as an optimization technique to solve the problem.

In this paper, we suggest an AS algorithm to find the optimal hydraulic system structure by choosing the appropriate hydraulic pipelines (technology of a hydraulic system element) from a list of available products in hydraulic market for each type of hydraulic pipeline. In practice, a variety of products are in fact available and each technology is characterized by its capacity, reliability and cost. Our objective is to select the optimal combination of hydraulic pipelines elements used in system for all elements corresponding to the minimal total cost subject to the requirement of meeting the water demand with the desirable level of reliability. The AS algorithm is inspired from nature like others meta-heuristic, e.g; simulated annealing, genetic algorithm, evolutionary strategy, and tabu search. The AS allows each component to contain elements with different technologies. To evaluate the reliability for arbitrary series-parallel hydraulic system structure, a fast procedure is developed which is based on universal generating function (UGF) [4] [8].

The rest of the paper is organized as follows. Section 2 of the paper consists of a general description of model used and a formulation of problem. In section 3 , we describe the reliability estimation method using the UGF technique. Section 4, describes the basic AS approach and its adaptation to the problem. In section 5, an illustrative example is represented. Conclusions are drawn in section 6.

\section{SUBJECT}

Let us consider a system containing $n$ hydraulic pipelines connected in series as sketched in (Fig. 1).

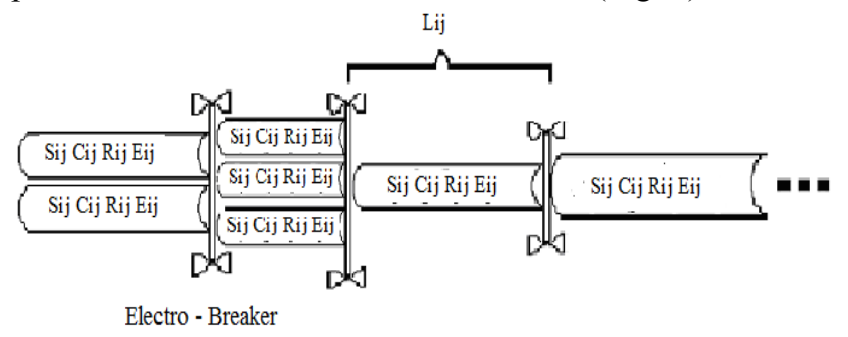

Fig. 1 Hydraulic series-parallel structure

Every component of type $i=1, \ldots, n$ contains a number of hydraulic pipelines. All the hydraulic pipelines of type $i$ belonging to different technologies are connected in mashed structure. A multi-choice of hydraulic pipelines and technologies will be adopted for each given system element. Each technology available in market has different costs, reliabilities, debit (section) and nominal capacities. A vector of parameters $C_{i_{v_{i}}}, R_{i_{v_{i}}}, E_{i_{v_{i}}}, S_{i_{v_{i}}}$ can be specified for each technology $v$ of element of type $i$. The hydraulic structure of system element $i$ is defined by the numbers of series and parallel hydraulic pipelines of each technology $k_{i v}$ for $1 \leq v \leq V_{i}$ where $V_{i}$ is the total number of technologies available of element of type $i$. The entire system structure is defined by a vector $k_{i}=\left\{k_{i v_{i}}\right\}$ $\left(1 \leq i \leq n, 1 \leq v \leq V_{i}\right)$ and the total cost of the system for given set $k_{1}, k_{2}, \ldots, k_{n}$ is formulated as follows:

$C=\sum_{i=1}^{n} \sum_{v=1}^{V_{i}} k_{i v} C_{i v}$

Usually in electrical power energy, the loss of load probability index (LOLP) is used for reliability estimation [5]. This index measure the probability that the load demand will not be meet. Generally the load demand is represented by discrete random curve. If the time period of load is the set of $M$ intervals, with duration $T_{j}$ 
$(j=1, \ldots, M)$, and each demand level duration, the LOLP is calculated as follows:

$$
L O L P=\frac{1}{\sum_{j=1}^{M} T_{j}} \sum_{j=1}^{M} P\left(E_{s}<d_{j}\right) T_{j}
$$

Where $P\left(E_{s}<d_{j}\right)$ represents the probability that the total system capacity $E_{s}$ is lower than the demand level $d_{j}$. All capacities production and demand are defined as a percentage of their total nominal value. The cumulative load curve is represented by vectors $d=\left\{d_{i}\right\}$ and $T=\left\{T_{j}\right\}$ who is known for every power system.

The measure of reliability system is defined by $R$ index in reference [2], given by the expression $R=1-L O L P$. This index will be compared and must be not less than some preliminarily specified level $R_{0}$.

The problem of hydraulic distribution system reliability optimization can be formulated as follow:

Find the hydraulic distribution system configuration $k_{1}, k_{2}, \ldots, k_{n}$ that provides the minimum total cost under reliability constraint. This problem can be started as below:

Minimise

$$
C=\sum_{i=1}^{n} \sum_{v=1}^{V_{i}} k_{i v} C_{i v}
$$

Subject To

$$
R\left(d, t, k_{1}, k_{2}, \ldots, k_{n}\right) \geq R_{0}
$$

\section{METHODS}

The problem defined above is one of combinatorial optimization problem, it is necessary to enumerate a huge number of possible system states. Thus, it is required to use an effective and fast procedure for structure reliability estimation. As shown above, the main problem is to evaluate the index $R$ for arbitrary series and parallel system. The probability that the total capacity of the hydraulic system is not less than a specific load water demand leveld must be calculated as:

$$
R(d)=P\left\{E_{s} \geq d\right\}=1-P\left\{E_{s}<d\right\}
$$

The procedure used to estimate this index is based on a modern mathematical technique: the UGF (or u-transform) technique in [9] [10] [11].This method was first applied to real power system reliability assessment and optimization in [12] [13], and represent an extension of ordinary moment generating function [14]. The UGF, in our case, of a discrete variable $E$ is defined as a polynomial

$$
u(z)=\sum_{j=1}^{J} P_{j} z^{e_{j}}
$$

where the discrete random variable $E$ has $J$ possible values and $P_{j}$ is the probability that $E$ is equal to $e_{j}$. Under consideration if only the elements with total failures are considered. For instance for each element of type $i$ and technology $v$ has reliability $R_{i v_{i}}$ and nominal capacity $E_{i v_{i}}$, then we denote by:
$P\left(E=E_{s}\right)=R_{i v}$ and $P(E=0)=1-R_{i v}$.

The UGF can be defined of such an element has only two terms as:

$u_{i v}=\left(1-R_{i v}\right) z^{0}+R_{i v} z^{E_{i v}}$

A brief overview on UGF method with respect to its applications for multi-states system (MSS) which has a finite number of states, there can be $H$ different levels of output performance at each time $t$ : $E(t) \in E=\left\{E_{h}, 1 \leq h \leq H\right\}$ and the system output performance can be defined by two finite vectors $E$ and $p=\left\{p_{h}(t)\right\}=\operatorname{Pr}\left\{E(t)=E_{h}\right\} 1 \leq h \leq H$, here the UGF, represented by the polynomial $u(z)$ can define all the MSS output performance, i.e. it represent all the possible states of the system by relating the probability of each state to performance of MSS in that state in the form:

$u_{M S S}(t, z)=\sum_{h=1}^{H} p_{h}(t) z^{E_{h}}$

Having the MSS output performance, the system reliability for arbitrary time $t$ and demand $d$ can be obtained using the following operator $\Omega_{A}$

$$
\begin{aligned}
R(t, d) & =\Omega_{A}\left(u_{M S S}(t, z), d\right) \\
& =\Omega_{A}\left(\sum_{h=1}^{\mathrm{H}} p_{h}(t) z^{E_{h}}, d\right) \\
& =\sum_{h=1}^{\mathrm{H}} p_{h}(t) \alpha\left(E_{h}-d\right)
\end{aligned}
$$

where

$$
\alpha(x)= \begin{cases}1, & x \geq 0 \\ 0, & x<0\end{cases}
$$

More explicitly, the probability that the total capacity of the power system is not less then a specified load level demand, and can be written as fellows:

$$
P\{E \geq d\}=\Omega\left(u(z) z^{-d}\right)
$$

Where $\Omega$ is a distributive operator defined by the following expression:

$$
\Omega\left(p z^{e-d}\right)=\left\{\begin{array}{lll}
p, & \text { if } & e \geq d \\
0, & \text { if } & e<d
\end{array}\right.
$$

And

$$
\Omega\left(\sum_{j=1}^{J} p z^{e_{j}-d}\right)=\sum_{j=1}^{J} \Omega\left(p z^{e_{j}-d}\right)
$$

For power system component containing $n$ elements connected in different ways, in parallel case, the total capacity is equal to the sum of capacities of all its elements. Therefore, the u-function can be calculated by using the $\Gamma$ operator: 
$u_{s}(z)=\Gamma\left(u_{1}(z), \ldots, u_{n}(z)\right)=\prod_{i=1}^{n} u_{i}(z)$

Where

$\Gamma\left(e_{1}, \ldots, e_{n}\right)=\sum_{i=1}^{n} e_{i}$ so that

$$
\begin{aligned}
\Gamma\left(u_{1}(z), u_{2}(z)\right) & =\Gamma\left(\sum_{i=1}^{n} p_{i} z^{a_{i}}, \sum_{j=1}^{m} q_{j} z^{b_{j}}\right) \\
& =\sum_{i=1}^{n} \sum_{j=1}^{m} p_{i} q_{j} z^{a_{i}+b_{j}}
\end{aligned}
$$

One can see that the $\Gamma$ operator is simply a product of polynomials representing the individual $\mathrm{u}$-functions. Other case if the system contains elements connected in series, the level of functioning is determined by the worst state observed for any one of its elements, and the worst state becomes the bottleneck of the system. Therefore, this element defines the total system capacity. To calculate the u-function for system containing $m$ elements connected in series, the operator $\beta$ should be used:

$$
u_{s}(z)=\beta\left(u_{1}(z), \ldots, u_{m}(z)\right)
$$

For which

$$
\beta\left(e_{1}, \ldots, e_{m}\right)=\min \left\{e_{1}, \ldots, e_{m}\right\}
$$

so that

$$
\begin{aligned}
\beta\left(u_{1}(z), u_{2}(z)\right) & =\beta\left(\sum_{i=1}^{n} p_{i} z^{a_{i}}, \sum_{j=1}^{m} q_{j} z^{b_{j}}\right) \\
& =\sum_{i=1}^{n} \sum_{j=1}^{m} p_{i} q_{j} z^{\min \left(a_{i}, b_{j}\right)}
\end{aligned}
$$

The MSS reliability was introduced as $P\{E \geq d\}$ after enough time has passed for this probability become constant.

\subsection{Example}

Consider for instance, two hydraulic power system pumps with nominal capacity $80 \mathrm{~L} / \mathrm{s}$. In the first hydraulic pump some types of failures appear and require the capacity to be reduced to $50 \mathrm{~L} / \mathrm{s}$ and others lead to the complete pump outage. In the second one some types of failures require the capacity to be reduced to $60 \mathrm{~L} / \mathrm{s}$, others lead to $40 \mathrm{~L} / \mathrm{s}$ and some type lead a complete pump outage.

So, there are three possible relative capacity levels that characterize the performance of the first hydraulic pump,

$E_{1}^{1}=\frac{0}{80}=0.0, E_{2}^{1}=\frac{50}{80}=0.62, E_{3}^{1}=\frac{80}{80}=1.0$

The corresponding steady state probabilities are:

$p_{1}^{1}=0.1, p_{2}^{1}=0.6, p_{3}^{1}=0.3$
For the first hydraulic pump. Now we can find the reliability for both MSS for the required capacity water level demand is $50 \mathrm{~L} / \mathrm{s}(d=0.5)$. The UGF system according the equation $[6]$ is:

$$
\begin{aligned}
u_{M S S}^{1}(z) & =p_{1}^{1} z^{E_{1}^{1}}+p_{2}^{1} z^{E_{2}^{1}}+p_{3}^{1} z^{E_{3}^{1}}= \\
& =0.1+0.6 z^{0.6}+0.3 z^{1.0}
\end{aligned}
$$

The MSS stationary reliability for water level demand $d=0.5$ is :

$$
R_{M S S}^{1}(d)=R_{M S S}^{1}(0.5)=\sum_{E_{h}^{1} \geq d} P_{h}=0.62+0.28=0.9
$$

Four relative capacity levels characterize the performance of the second hydraulic pump,

$E_{1}^{2}=\frac{0}{80}=0.0, E_{2}^{2}=\frac{60}{80}=0.75, E_{3}^{2}=\frac{40}{80}=0.5, E_{4}^{2}=\frac{80}{80}=1.0$

The corresponding steady state probabilities are:

$p_{1}^{2}=0.05, p_{2}^{2}=0.25, p_{3}^{2}=0.3, p_{4}^{2}=0.4$

The UGF system is :

$$
\begin{aligned}
u_{M S S}^{2}(z) & =p_{1}^{2} z^{E_{1}^{2}}+p_{2}^{2} z^{E_{2}^{2}}+p_{3}^{2} z^{E_{3}^{2}}+p_{4}^{2} z^{E_{4}^{2}}= \\
& =0.05+0.25 z^{0.4}+0.3 z^{0.8}+0.4 z^{1.0}
\end{aligned}
$$

The MSS stationary reliability for level demand $d=0.5$ is :

$$
\begin{aligned}
& R_{M S S}^{1}(d)=R_{M S S}^{1}(0.5)=\sum_{E_{h}^{1} \geq d} P_{h}=0.62+0.28=0.9 \\
& R_{M S S}^{2}(d)=R_{M S S}^{2}(0.5)=\sum_{E_{h}^{1} \geq d} P_{h}=0.8+0.4=0.7
\end{aligned}
$$

Note that since obtained reliability indices, the first hydraulic system performs better than the second, contrary when the expected debit is considered. Really, analyze of this problem is very complex and consist of a wild number of hydraulic pipelines connected in different ways. To obtain the corresponding UGF, we must develop some rules in order to determine the system UGF based on the individual u-function of its hydraulic pipelines. The system structure is mashed, to obtain the latter of an elements containing a number of hydraulic pipelines, some composition operators are used. These operators determine the component $\mathrm{u}$-function expressed as polynomial equation (5), for a group of two different elements of type $i$ take the form

$$
\begin{aligned}
\Psi_{\Omega}\left(u_{1}(z), u_{2}(z)\right) & =\Psi_{\Omega}\left[\sum_{i=1}^{I} P_{1 i} z^{e_{1 i}} ; \sum_{j=1}^{J} P_{2 j} z^{e_{2 j}}=\right. \\
& =\sum_{i=1}^{I} \sum_{j=1}^{J} P_{1 i} P_{2 j} z
\end{aligned}
$$


Where $u_{1}(z), u_{2}(z)$ are the individual $\mathrm{u}$-function of elements and $\Omega$ is composition operators expresses the entire performance of a system consisting of different performance in terms of individual performance of the hydraulic pipelines. The definition of the function $\Omega$ strictly depends of the type of connection between hydraulic pipelines, on physical nature of system performance measure and in the reliability diagram sense, i.e. on the topology of hydraulic system structure. Also, for series connection, the level of functioning is determined by the worst state observed for any one of its hydraulic pipelines, while for parallel connection it is determined by the best state. However, such an approach is not applicable for the majority of real-world systems. For a hydraulic power system distribution containing a pair of hydraulic pipelines connected in parallel, the total capacity is equal the sum of capacities. Therefore, its u-function can be calculated using the $\Omega$ operator:

$$
\Omega\left(e_{1}, e_{2}\right)=\max \left(e_{1}, e_{2}\right)=e_{1}+e_{2}
$$

And for a pair of hydraulic pipelines connected in series the $\mathrm{u}$-function can be calculated as:

$$
\Omega\left(e_{1}, e_{2}\right)=\min \left(e_{1}, e_{2}\right)
$$

\section{$\Psi$ operators were determined in [13]}

\subsection{The ant colony optimization approach}

The problem formulated in this paper is a complicated combinatorial optimization problem. The total number of different solutions to be examined is very large, even for rather small problems. An exhaustive examination of the enormous number of possible solutions is not feasible given reasonable time limitations. Thus, because of the search space size of the ROP for MSS, a new meta-heuristic is developed in this section. This meta-heuristic consists in an adaptation of the ant colony optimization method.

\subsection{The ACO principle}

Recently, (Dorigo, Maniezzo and Colorni, 1996) introduced a new approach to optimization problems derived from the study of any colonies [7], called "Ant System". Their system inspired by the work of real ant colonies that exhibit the highly structured behaviour [1]. Ants lay down in some quantity an aromatic substance, known as pheromone, in their way to food. An ant chooses a specific path in correlation with the intensity of the pheromone. The pheromone trail evaporates over time if no more pheromone in laid down by others ants, therefore the best paths has more intensive pheromone and higher probability to be chosen. This simple behaviour explains why ants are able to adjust to changes in the environment, such as new obstacles interrupting the currently shortest path.

Artificial ants used in ant system are agents with very simple basic capabilities mimic the behaviour of real ants to some extent. This approach provides algorithms called ant algorithms. The Ant System approach associates pheromone trails to features of the solutions of a combinatorial problem, which can be seen as a kind of adaptive memory of the previous solutions. Solutions are iteratively constructed in a randomized heuristic fashion biased by the pheromone trails, left by the previous ants. The pheromone trails, $\tau_{i j}$, are updated after the construction of a solution, enforcing that the best features will have a more intensive pheromone. An Ant algorithm presents the following characteristics. It is a natural algorithm since it is based on the behaviour of ants in establishing paths from their colony to feeding sources and back. It is parallel and distributed since it concerns a population of agents moving simultaneously, independently and without supervisor. It is cooperative since each agent chooses a path on the basis of the information, pheromone trails, laid by the other agents with have previously selected the same path. It is versatile that can be applied to similar versions the same problem. It is robust that it can be applied with minimal changes to other combinatorial optimization problems. The solution of the travelling salesman problem (TSP) was one of the first applications of ACO.

Various extensions to the basic TSP algorithm were proposed, notably by Dorigo and Gambardella (Dorigo and Gambardella, 1997a). The improvements include three main aspects: the state transition rule provides a direct way to balance between exploration of new edges and exploitation of a priori and accumulated knowledge about the problem, the global updating rule is applied only to edges which belong to the best ant tour and while ants construct solution, a local pheromone updating rule is applied. These extensions have been included in the algorithm proposed in this paper.

\subsection{ACO_based solution approach}

In our reliability optimization problem, we have to select the best combination of hydraulic pipelines to minimize the total cost given a reliability constraint. The hydraulic pipelines can be chosen in any combination from the available hydraulic pipelines. Hydraulic pipelines are characterized by their reliability, capacity, debit and cost. This problem can be represented by a graph (figure 2) in which the set of nodes comprises the set of subsystems and the set of available hydraulic pipelines (i.e. $\max \left(M_{j}\right), j=$ $1 . . n$ ) with a set of connections partially connect the graph (i.e. each subsystem is connected only to its available hydraulic pipelines). An additional node (blank node) is connected to each subsystem.

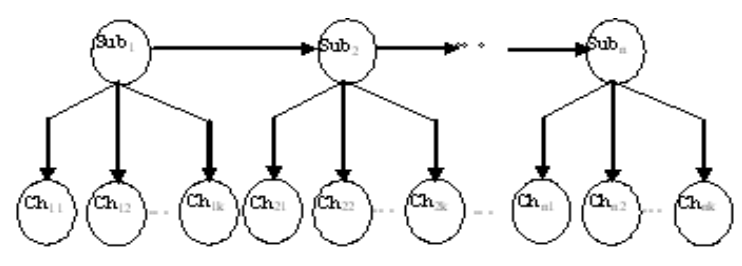

Fig 2 Definition of a series-parallel system with tree subsystems into a graph 
In figure 2, a series-parallel system is illustrated where the first and the second subsystem are connected respectively to their 3 and 2 available hydraulic pipelines. The nodes $c p_{i 3}$ and $c p_{i 4}$, represent the blank hydraulic pipelines of the two subsystems. At each step of the construction process, an ant uses problem-specific heuristic information, denoted by $\eta_{i j}$ to choose the optimal number of hydraulic pipelines in each subsystem. An imaginary heuristic information is associated to each blank node. These new factors allow us to limit the search surfaces (i.e. tuning factors). An ant positioned on subsystem $i$ chooses a hydraulic pipelines $\mathrm{j}$ by applying the rule given by:

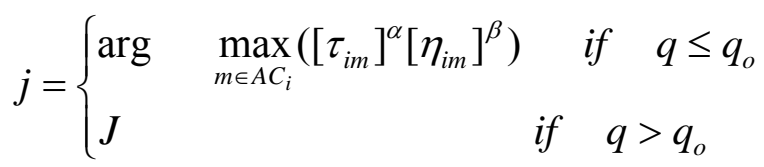

and $J$ is chosen according to the probability:

$$
p_{i j}=\left\{\begin{array}{l}
\frac{\left[\tau_{i j}\right]^{\alpha}\left[\eta_{i j}\right]^{\beta}}{\sum_{m \in A C_{i}}\left[\tau_{i m}\right]^{\alpha}\left[\eta_{i m}\right]^{\beta}} \quad \text { if } \quad j \in A C_{i} \\
0 \quad \text { otherwise }
\end{array}\right.
$$

$\alpha$ : The relative importance of the trail.

$\beta$ : The relative importance of the heuristic information $\eta_{i j}$.

$A C_{i}$ : The set of available components choices for subsystem $i$.

$q$ : Random number uniformly generated between 0 and 1.

The heuristic information used is: $\eta_{i j}=1 /\left(1+c_{i j}\right)$ where $c_{i j}$ represents the associated cost of hydraulic pipelines $j$ for subsystem $i$. A "tuning" factor $t_{i}=\eta_{i j}=1 /\left(1+c_{i(M i+1)}\right)$ is associated to blank component $\left(M_{i}+1\right)$ of subsystem $i$. The parameter $q_{o}$ determines the relative importance of exploitation versus exploration: every time an ant in subsystem $i$ have to choose a component $j$, it samples a random number $0 \leq q \leq 1$. If $q \leq q_{o}$ then the best edge, according to (12), is chosen (exploitation), otherwise an edge is chosen according to (13) (biased exploration).

The pheromone update consists of two phases: local and global updating. While building a solution of the problem, ants choose components and change the pheromone level on subsystem-component edges. This local trail update is introduced to avoid premature convergence and effects a temporary reduction in the quantity of pheromone for a given subsystem-component edge so as to discourage the next ant from choosing the same component during the same cycle. The local updating is given by:

$$
\tau_{i j}^{n e w}=(1-\rho) \tau_{i j}^{o l d}+\rho \tau_{o}
$$

Where $\rho$ is a coefficient such that $(1-\rho)$ represents the evaporation of trail and $\tau_{o}$ is an initial value of trail intensity. It is initialized to the value $\left(n . T C_{n n}\right)^{-1}$ with $n$ is the size of the problem (i.e. number of subsystem and total number of available components) and $T C_{n n}$ is the result of a solution obtained through some simple heuristic.

After all ants have constructed a complete system, the pheromone trail is then updated at the end of a cycle (i.e. global updating), but only for the best solution found. This choice, together with the use of the pseudo-randomproportional rule given by (15) and (16), is intended to make the search more directed: ants search in a neighbourhood of the best solution found up to the current iteration of the algorithm. The pheromone level is updated by applying the following global updating rule:

$\tau_{i j}^{\text {new }}=(1-\rho) \tau_{i j}^{\text {old }}+\rho \Delta \tau_{i j}$

$\Delta \tau_{i j}=\left\{\begin{array}{lr}\frac{1}{T C_{\text {best }}} & \text { if }(i, j) \in \text { best } \\ 0 & \text { otherwise }\end{array}\right.$

\subsection{The algorithm}

An ant-cycle algorithm is stated as follows. At time zero an initialization phase takes place during wish $\mathrm{NbAnt}$ ants select components in each subsystem according to the Pseudo-random-proportional transition rule given by (15) and (16). When an ant selects a component, a local update is made to the trail for that subsystem-component edge according to equation (14). In this equation, $\rho$ is a parameter that determines the rate of reduction of the pheromone level? The pheromone reduction is small but sufficient to lower the attractiveness of precedent subsystem-component edge. At the end of a cycle, for each ant $\mathrm{k}$, the value of the system's reliability $R_{k}$ and the total cost $T C_{k}$ are computed. The best feasible solution found by ants (i.e. total cost and assignments) is saved. The pheromone trail is then updated for the best solution obtained according to (15) and (16). This process is iterated until the tour counter reaches the maximum number of cycles $N C_{\max }$ or all ants make the same tour (stagnation behavior).

The followings are formal description of the algorithm.

1. Set NC: $=0$ (NC: cycle counter)

For every edge $(\mathrm{i}, \mathrm{j})$ set an initial value $\tau_{\mathrm{ij}}(0)=\tau_{\mathrm{o}}$

2. For $\mathrm{k}=1$ to $\mathrm{NbAnt}$ do

For $\mathrm{i}=1$ to $\mathrm{NbSubSystem} \mathrm{do}$ pipes) do

For $\mathrm{j}=1$ to MaxComponents (Max of hydraulic

Choose a component, including blanks, according to (1) and (2).

Local update of pheromone trail for chosen subsystemcomponent (hydraulic pipes) edge (i,j):

$$
\tau_{i j}^{n e w}=(1-\rho) \tau_{i j}^{o l d}+\rho \tau_{o}
$$

End For 
End For

3. Calculate $R_{k}$ (system reliability for each ant)

Calculate the total cost for each ant $\mathrm{TC}_{\mathrm{k}}$

Update the best found feasible solution

4. Global update of pheromone trail:

For each edge $(i, j) \in$ best feasible solution, update the pheromone trail according to:

$\tau_{i j}^{\text {new }}=(1-\rho) \tau_{i j}^{\text {old }}+\rho \Delta \tau_{i j}$

$\Delta \tau_{i j}=\left\{\begin{array}{lr}\frac{1}{T C_{\text {best }}} & \text { if }(i, j) \in \text { best } \\ 0 & \text { otherwise }\end{array}\right.$

End For

5. cycle $=$ cycle +1

6. if $\left(\mathrm{NC}<\mathrm{NC}_{\max }\right)$ and (not stagnation behavior)

Then

Go to step 2

Else

Print the best feasible solution and components selection.

Stop.

\section{RESULTS}

In order to illustrate the proposed ant colony algorithm, a numerical example is solved by use of the data given in Table 1. Each element of the sub-system is considered as a unit with total failures. Table 2 contains the data of cumulative demand.

The maximum numbers of components $p_{\max }$ in parallel are set to $(8,6,5,10)$. The number of ants used to find the best solution is 100 . The simulation results depend greatly on the values of the coefficients $\alpha$ and $\beta$. Different $t_{i}$ values (tuning factors associated to blank components) were tested and shown to influence greatly the algorithm. The best found values of $t_{i}$ are $\left(t_{1}=-0.13, t_{2}=-0.04, t_{3}=2.3, t_{4}=-0.35\right.$, $\left.t_{5}=0.35\right)$. Several simulations are made for $\alpha=1$ and $\beta=2$ and the best solution is obtained in 400 cycle (Figure 2). Table 3 presents the obtained configuration.

Table 1 Data of available different hydraulic pipes Technologies

\begin{tabular}{|c|c|c|c|c|}
\hline $\begin{array}{c}\text { Basic } \\
\text { Compone } \\
\text { nts } \\
\#\end{array}$ & $\begin{array}{c}\text { Different } \\
\text { Technologies } \\
\text { of Hydraulic Pipe } \\
\#\end{array}$ & $\begin{array}{c}\text { Availability } \\
\text { R }\end{array}$ & $\begin{array}{c}\text { Cost } \\
\text { C }\end{array}$ & $\begin{array}{c}\text { Capacity } \\
\text { G }\end{array}$ \\
\hline & 1 & 0.980 & 0.590 & 120 \\
$\mathbf{1}$ & 2 & 0.977 & 0.535 & 100 \\
& 3 & 0.982 & 0.470 & 85 \\
& 4 & 0.978 & 0.420 & 85 \\
\hline & 1 & 0.995 & 0.205 & 100 \\
& 2 & 0.996 & 0.189 & 92 \\
& 3 & 0.997 & 0.091 & 53 \\
& 4 & 0.997 & 0.056 & 28 \\
3 & 5 & 0.998 & 0.042 & 21 \\
\hline & 1 & 0.971 & 7.525 & 100 \\
& 2 & 0.973 & 4.720 & 60 \\
& 3 & 0.971 & 3.590 & 40 \\
\hline
\end{tabular}

Table 2 Parameters of the water demand cuve

\begin{tabular}{|c|c|c|c|c|}
\hline $\begin{array}{c}\text { Capacity Water } \\
\text { Demand level (\%) }\end{array}$ & $\mathbf{1 0 0}$ & $\mathbf{8 0}$ & $\mathbf{5 0}$ & $\mathbf{2 0}$ \\
\hline Duration (h) & 4203 & 788 & 1228 & 2536 \\
\hline Probability & 0.479 & 0.089 & 0.140 & 0.289 \\
\hline
\end{tabular}

Table 3 Optimal solutions obtained by ant colony algorithm

\begin{tabular}{|c|c|c|c|}
\hline $\mathbf{R}_{0}$ & $\begin{array}{l}\text { Optimal Structure } \\
\text { Of Water network }\end{array}$ & $\begin{array}{c}\text { Computed } \\
\text { Availability } \\
\mathbf{R}\end{array}$ & $\begin{array}{c}\text { Computed } \\
\text { Cost } \\
\text { C (mI\$) } \\
\end{array}$ \\
\hline 0.975 & $\begin{array}{c}\text { Subsystem } \\
\mathbf{1 : 1 ( 4 )}-\mathbf{( 3 )} \\
\text { Subsystem } \\
\mathbf{2 : 1 ( 4 )}-\mathbf{3 ( 5 )} \\
\text { Subsystem } \\
\mathbf{3 : 1 ( 1 )}-\mathbf{3 ( 4 )}\end{array}$ & 0.978 & 04.88 \\
\hline
\end{tabular}

Table 3 shows the best optimal power hydraulic pipe structure obtained by the suggested ant colony for one desired reliability levels $A_{0}$ ( 0.975$)$. This latter illustrate the computed cost and availability index to the corresponding hydraulic pipe structure. In the ant algorithm a set of parameter values are tested. When the demand varies the best values corresponding to the merit structures are: $\alpha=5$, $\beta=1, \tau_{0}=0.5$ and $\rho=0.080$. The choice of these values affects strongly the solution. Since it is a heuristic method only near optimal solutions can be obtained.

To compare this meta-heuristic to the combinatorial one, the space searching is about $100 * 400$ cycles, but in combinatorial one is $10^{48}$. The Program was run on PC Intel. IV with $2.4 \mathrm{GHz}$. The time to find the best solution is $1 ' .05 "$. Not realistic in combinatorial method.

\section{CONCLUSION}

In this paper, we solve the power hydraulic pipe optimal structure which is a very interesting problem often reencountered in energy industry or manufacturing industry. It is formulated as redundancy optimization problem. The resolution of this problem uses a developing ant colony method. This new algorithm for choosing an optimal series-parallel power hydraulic pipe structure configuration is proposed which minimizes total investment cost subject to availability constraints. This algorithm seeks and selects hydraulic pipe technologies among a list of available products according to their availability, nominal capacity (performance) and cost. Also defines the number and the kind of series-parallel power hydraulic pipe to put in each subsystem when consumers' demand changes. The proposed method allows a practical way to solve wide instances of reliability optimization problem of multi-state systems without limitation on the diversity of hydraulic pipe technologies put in seriesparallel. A combination is used in this algorithm based on the universal moment generating function and an ACO algorithm. 


\section{ACKNOWLEDGMENTS}

My thanks for professor Zeblah Abdelkader, for having accompanied, encouraged, reviewed, commented and corrected the article.

\section{REFERENCES}

[1] LIANG, Y. C. - SMITH, A. E.: An ant colony approach to redundancy allocation, IEEE Transaction on reliability October (2001).

[2] LEVITIN, G. - LISNIASKI, A. - ELMAKIS, D.: Structure optimization of power system with different redundant cells, Elsevier Science S.A. (1996-1997).

[3] COIT, D. W. - SMITH, A. E.: Optimization approaches to the redundancy allocation problem for series-parallel systems, Proceeding of the fourth industrial engineering research conference (IERC) May, 1995.

[4] USHAKOV, I. A.: Optimal standby problems and a universal generating function, Sov. J. Compt. Syst. Sci. 25 (4) 1987, 79-82.

[5] BILlitON, R. - ALLAN, R.: Reliability of power systems, Pitman, London, 1984.

[6] USHAKOV, I. A. - HARRISON, A.: (Eds), Handbook of reliability engineering, Wiley and Sons, NY/Chichester/Toronto, 1994.

[7] DARIGO, M. - MANIEZZO, V. - COLORNI, A.: optimization by a colony of cooperating agents, IEEE Transaction on systems, Man, and cybernetic-part B, Vol. 26, No. 1, 1996, pp. 1-13.

[8] LEVITIN, G. - LISNIASKI, A. - BEN-HAIM, H. ELMAKIS, D.: Power system structure optimization subject to reliability constraints, Electric Power System research 39, 1996, 145-152.

[9] USHAKOV, I. A.: Universal generating function, Sov. J. Compt. Syst. Sci. 24 (5), (1986), 118-129.

[10] USHAKOV, I. A.: A Universal generating function, Sov. Journal, computer system science.1986; 24: 3749.

[11] USHAKOV, I. A.: Optimal standby problems and a universal generating function, Sov. Journal, computer system science. $1987 ; 25$ : 61-73.
[12] LEVITIN, G. - LISNIASKI, A. - BEN-HAIM, H. ELMAKIS, D.: Redundancy optimization for power station. Proceedings of the 10th International Conference of Israel Society for quality, Jurusalem, Israel, 1994: 313-318.

[13] LEVITIN, G. - LISNIASKI, A. - BEN-HAIM, H. ELMAKIS, D.: Redundancy optimization for seriesparallel multi-state system, IEEE Transaction on reliability 1998; 47(2) 165-172.

[14] ROSS, S. M.: Introduction to probability model, Academic Press, New York, 1993. Kolski C., Interfaces homme-machine, Paris, Hermès, 1997.

[15] NAWROCKI A.: Contribution à la modélisation des câbles monotorons par éléments finis, Thèse de doctorat, Université de Nantes, 1997.

Received March 8, 2018, accepted May 21, 2018

\section{BIOGRAPHIES}

Bouri Sihem was born in Ain Témouchent (Algeria), on August 4, 1976. She graduated the Faculty of Technology, Sidi Bel Abess (Algeria), in 1999. She received the PhD degree in electrical engineering from the the Faculty of Technology, Sidi Bel Abess (Algeria), in 2008. She is Professor at the Faculty of Technology, in Tlemcen (Algeria). Her research interests concern: power systems, reliability, Universal moment generating Function (UMGF).

Abdelhalim Benmansour was born on January 2, 1957 in Tlemcen (Algeria). He graduated from the University of Sciences and Technology of Algiers in Physics in 1979 and from the National School of Electrical Engineers (ENSIEG) of the National Polytechnic Institute (INPG) of Grenoble (France) in electrical engineering in 1982. He obtained his energetic magister in 1992 and his $\mathrm{PhD}$ in materials science in 2004 at the University of Tlemcen (Algeria). He is professor at the faculty of technology and director of the research unit on materials and renewable energies (URMER) at the University of Tlemcen (Algeria). His research interests include: photovoltaic cells, materials, renewable energy and water treatment. 